\title{
Phase-separation transition in one-dimensional driven models
}

\author{
Y. Kafri, ${ }^{1}$ E. Levine, ${ }^{1}$ D. Mukamel, ${ }^{1}$ G. M. Schütz,${ }^{2}$ and R. D. Willmann ${ }^{1,2}$ \\ ${ }^{1}$ Department of Physics of Complex Systems, Weizmann Institute of Science, Rehovot, Israel 76100 \\ ${ }^{2}$ Institut für Festkörperforschung, Forschungszentrum Jülich, 52425 Jülich, Germany
}

(Received 14 November 2002; revised manuscript received 26 June 2003; published 9 September 2003)

\begin{abstract}
A class of models of two-species driven diffusive systems which is shown to exhibit phase separation in $d=1$ dimensions is introduced. Unlike previously studied models exhibiting similar phenomena, here the relative density of the two species is fluctuating within the macroscopic domain of the phase separtated state. The nature of the phase transition from the homogeneous to the phase-separated state is discussed in view of a recently introduced criterion for phase separation in one-dimensional driven systems.
\end{abstract}

DOI: 10.1103/PhysRevE.68.035101

PACS number(s): 02.50.Ey, 05.60.-k, 05.20.-y, 64.75.+g

One-dimensional driven diffusive systems have attracted considerable attention in recent years [1]. It has been demonstrated in numerous studies that unlike systems in thermal equilibrium, certain driven diffusive models with local noisy dynamics do exhibit phenomena such as phase transitions and phase separation. More recently a criterion for the existence of phase separation in a class of driven onedimensional models has been introduced [2]. The criterion relates the existence of phase separation in a given model to the rate at which domains of various sizes exchange particles. Assuming that for a domain of length $n$ this rate is given by the steady-state current $J_{n}$ which flows through it, phase separation was suggested to exist only in the following cases: either the current vanishes in the thermodynamic limit,

$$
J_{n} \rightarrow 0 \quad \text { as } \quad n \rightarrow \infty \quad(\text { Case } A)
$$

or the behavior of the current for large domains is of the form

$$
J_{n} \sim J_{\infty}\left(1+b / n^{\sigma}\right) \quad(\text { Case } B),
$$

for either $\sigma<1$ and $b>0$ or for $\sigma=1$ and $b>2$.

The nature of the phase-separated states is rather different in the two cases. In case $A$ the phase-separated states were found to be of a rather simple nature, characterized by coexistence of pure domains, each consisting of a single type of particles. Thus, the particle density in the interior of a domain is nonfluctuating. Density fluctuations are limited to finite regions around the domain boundaries. Such steady states were termed strongly phase-separated. Moreover, in this case phase separation is expected to take place at any density, no matter how small. On the other hand, in case $B$ the phase-separated state is expected to be fluctuating in the bulk of macroscopic domains, as is normally expected in a noisy system. It exists only at high enough densities, while at low densities the system is homogeneous. This phase was termed condensed as the mechanism of the transition is similar to that of the Bose-Einstein condensation.

One-dimensional models shown so far to phase separate are of type $A$ [3-5], and thus they exhibit strong phase separation at any density. In these models more than one species of particles are involved. In a recent study by Arndt et al. (AHR) [4] an interesting two species driven model was introduced. It was suggested, based on numerical simulations, that the model exhibits a condensed phase-separated state, whereby the particle densities fluctuate in the interior of the coexisting domains and not just at the domain boundaries. In this state, a region with a high density of particles of both species coexists with a low-density region. Moreover, the model has nonvanishing currents even in the thermodynamic limit. As in equilibrium phase separation it has been suggested that this state exists only at sufficiently high densities. However, a subsequent exact solution of the model [6] shows that what numerically seems like a condensed state is in fact homogeneous, with a very large but finite correlation length. Further analysis of this model, in the light of the criterion suggested in Ref. [2] shows that the currents $J_{n}$ corresponding to the domains in this model are given by the form $B$, with $\sigma=1$ and $b=3 / 2$ [2]. Therefore, according to the criterion, no phase separation takes place.

Another example of a model which was suggested to exhibit phase separation into a fluctuating macroscopically inhomogeneous state is the two-lane model introduced by Korniss et al. [7]. While numerical studies of the model indicate that such a phase exists in the model, studies of the current $J_{n}$ of finite domains suggests that it is of type $B$ with $\sigma=1$ and $b \simeq 0.8$ [2], indicating, again, that no phase separation exists in this model. Thus the question of whether a phase separation of type $B$ exists remains an intriguing open question.

In this paper we introduce a class of models which are demonstrated to be of type $B$, with $\sigma=1$ and $b>2$. According to the criterion conjectured in Ref. [2] this class is expected to exhibit a phase transition to a phase-separated condensed state. Thus at high densities these models exhibit phase separation with nonvanishing currents in the thermodynamic limit, and bulk fluctuations which are not restricted to the vicinity of the domain boundaries. To the best of our knowledge, this is the first example of a density driven transition of this type in one-dimensional driven systems.

We now define this class of models in detail. We consider a one-dimensional ring with $L$ sites. Each site $i$ can be either vacant $(0)$ or occupied by a positive $(+)$ or a negative ( - ) particle (or charge). Positive particles are driven to the right while negative particles are driven to the left. In addition to the hard-core repulsion, particles are subject to shortrange interactions. These interactions are "ferromagnetic," in the sense that particles of the same kind attract each other. 


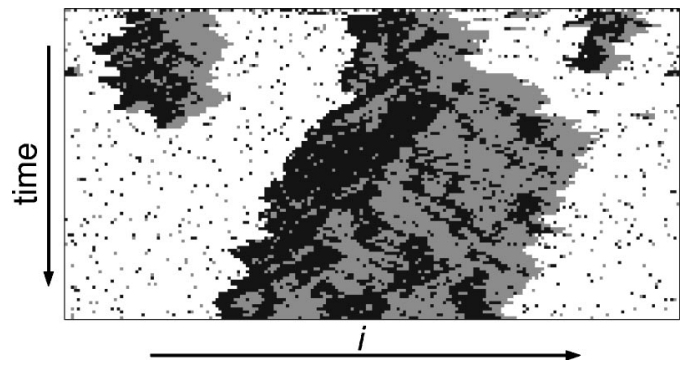

FIG. 1. Evolution of a random initial configuration of model (3) with nearest-neighbor interactions, on a ring of 200 sites. Here $\epsilon$ $=0.9, \alpha=2$, and the particle density is $\rho=0.5$. Positive particles are colored black and negative particles are colored gray. One hundred snapshots of the system are shown at intervals of 100 Monte Carlo sweeps.

The dynamics conserves the number of particles of each species, $N_{+}$and $N_{-}$. The total density of particles in the system is $\rho=\left(N_{+}+N_{-}\right) / L$. The model is defined by a randomsequential local dynamics, whereby a pair of nearestneighbor sites is selected at random, and the particles are exchanged with the following rates:

$$
\begin{aligned}
& +-\rightarrow-+ \text { with rate } 1-\Delta H, \\
& +0 \rightarrow 0+\text { with rate } \alpha, \\
& 0-\rightarrow-0 \text { with rate } \alpha .
\end{aligned}
$$

Here $\Delta H$ is the difference in the ferromagnetic interactions between the final and the initial configurations. We begin by considering a model with only nearest-neighbor interactions,

$$
H=-\epsilon / 4 \sum_{i} s_{i} s_{i+1}
$$

Here $s_{i}=+1(-1)$ if site $i$ is occupied by a $+(-)$ particle and $s_{i}=0$ if site $i$ is vacant. To ensure positive transition rates we take $0 \leqslant \epsilon<1$. The model is a generalization of the Katz-Lebowitz-Spohn (KLS) model, introduced in Ref. [8] and studied in detail in Ref. [9], in which the lattice is fully occupied by charges and no vacancies exist. In this paper we consider the case where the number of positive and negative particles is equal, $N_{+}=N_{-}$.

We will demonstrate that for a certain range of the parameters defining the dynamics, namely, for $\epsilon>0.8$ and sufficiently large $\alpha$ (to be discussed below), a phase separation transition occurs as the density $\rho$ is increased above a critical density $\rho_{c}$. In the phase-separated state a macroscopic domain, composed of positive and negative particles, coexists with a fluid phase, which consists of small domains of particles (of both charges) separated by vacancies. Typical configurations obtained during the time evolution of the model starting from a random initial configuration are given in Fig. 1. This figure suggests that a coarsening process takes place, leading to a phase-separated state as described above. However, this by itself cannot be interpreted as a demonstration of phase separation in these models. The reason is that this behavior may very well be a result of a very large but finite correlation length, as is the case in the AHR $[4,6]$ and the two-lane [7] models discussed above [10]. We thus apply the criterion introduced in [2] in order to analyze the possible existence of phase separation in this model.

To this end we define a domain as an uninterrupted sequence of positive and negative particles bounded by vacancies from both ends. The current $J_{n}$ corresponding to such a domain of length $n$ may thus be determined by studying an open chain, fully occupied by positive and negative particles, with entrance and exit rates $\alpha$. This is just the onedimensional KLS model on an open chain. Phase separation is expected to take place only for sufficiently large $\alpha$. We consider $\alpha$ such that the system is in its maximal current state, whereby $J_{\infty}$ assumes its maximum possible value and is independent of $\alpha$.

To evaluate $J_{n}$ we first consider the KLS model on a ring of $n$ sites with no vacancies. We then extend these results to study the behavior of an open chain. Since we are interested in the maximal current phase we consider equal number of positive and negative particles $n_{+}=n_{-}=n / 2$. It can be shown, as was done for the noisy Burger's equation $[12,13]$, that under quite general conditions, to be discussed below, the current $J_{n}$ takes the following form for large $n$ :

$$
J_{n}=J_{\infty}\left(1-\frac{\lambda \kappa}{2 J_{\infty}} \frac{1}{n}\right) .
$$

Here $\lambda=\partial^{2} J_{\infty} / \partial \rho_{+}^{2}$ is the second derivative of the current with respect to the density of positive particles $\rho_{+}$in the system. The compressibility analog $\kappa$ is defined by $\kappa$ $=\lim _{n \rightarrow \infty} n^{-1}\left(\left\langle n_{+}^{2}\right\rangle-\left\langle n_{+}\right\rangle^{2}\right)$, calculated within a grand canonical ensemble, as explained below. This can be demonstrated by considering the current $J_{n}\left(n_{+}\right)$for charge densities close to $n_{+}=n_{-}=n / 2$. Expanding $J_{n}\left(n_{+}\right)$in powers of $\Delta n_{+}=n_{+}-n / 2$ one has

$$
J_{n}\left(n_{+}\right)=J_{n}(n / 2)+J_{n}^{\prime} \Delta n_{+}+\frac{1}{2} J_{n}^{\prime \prime}\left(\Delta n_{+}\right)^{2},
$$

where the derivatives $J_{n}^{\prime}$ and $J_{n}^{\prime \prime}$ are taken with respect to $n_{+}$ and evaluated at $n / 2$. We average (6) over $n_{+}$with the steady-state weights of a grand canonical ensemble. This is done by introducing a chemical potential $\mu$ which ensures that the average density satisfies $\left\langle n_{+}\right\rangle=n / 2$. We find

$$
\left\langle J_{n}\left(n_{+}\right)\right\rangle_{\mu}=J_{n}(n / 2)+\frac{1}{2} J_{n}^{\prime \prime}\left\langle\left(\Delta n_{+}\right)^{2}\right\rangle_{\mu} .
$$

Noting that $\left\langle J_{n}\left(n_{+}\right)\right\rangle_{\mu}$ is $J_{\infty}$ in the $n \rightarrow \infty$ limit, and $J_{n}(n / 2)$ is just $J_{n}$, Eq. (5) is obtained. Here we made use of the fact that finite-size corrections to $\left\langle J_{n}\left(n_{+}\right)\right\rangle_{\mu}$, resulting from the next to leading eigenvalue of the transfer matrix of the steady-state distribution, are exponentially small in $n$ and may thus be neglected. The result of Eq. (5) is rather general and is independent of the exact form of the steady-state particle distribution. This is provided that the weights of the microscopic configurations are local and thus the density and chemical potential ensembles are equivalent.

In fact, an alternative way to derive (5) is to consider the correspondence between the driven lattice-gas models and the noisy Burger's equation or the Kardar-Parisi-Zhang 


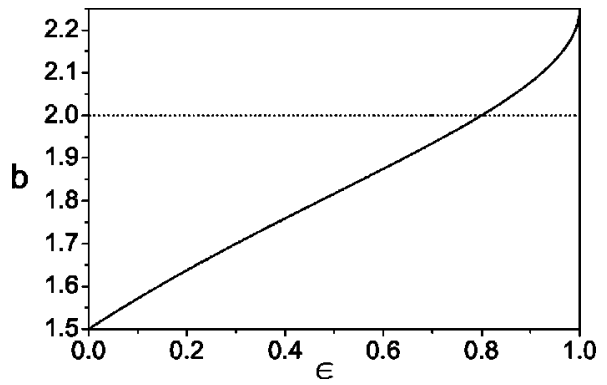

FIG. 2. The coefficient $b(\epsilon)$, Eq. (10).

(KPZ) equation for interface growth in $1+1$ dimensions [11]. In these models $J_{n}$ corresponds to the growth velocity of the interface. Equation (5) has been derived in Refs. $[12,13]$, where $\lambda$ is the coefficient of the nonlinear term in the KPZ equation. The equivalence of the two alternative approaches relies on the fact that both $\kappa$ and $\lambda$ are invariant under renormalization transformations.

Result (5) can be used to evaluate $J_{n}$ for the KLS model. It has been shown $[8,10]$ that for a ring geometry the steadystate weight of a configuration $\left\{\tau_{i}\right\}$ is

$$
P\left(\left\{\tau_{i}\right\}\right)=e^{-\beta \mathcal{H}}, \quad \mathcal{H}=-\sum_{i=1}^{n} \tau_{i} \tau_{i+1}-\mu \sum_{i=1}^{n} \tau_{i},
$$

with $\tau_{i}= \pm 1$ for positive and negative charges, respectively, $e^{4 \beta}=(1-\epsilon) /(1+\epsilon)$, and $\mu$ serves as a chemical potential which controls the density of, say, the positive particles. The chemical potential $\mu$ vanishes for the case $n_{+}=n_{-}$. Using (8) expressions for $\kappa(\epsilon)$ and $J_{\infty}(\epsilon)$ of this model have been obtained in Ref. [10].

We now consider the KLS model in an open chain, which is the relevant geometry in applying the phase-separation criterion. It has been argued [12] that the finite-size correction to the current of an open chain is given by the corresponding correction in a ring geometry, up to a universal multiplicative constant $c$ which depends only on the boundary conditions. In the maximal current phase, $c$ was found to be $3 / 2$. Thus the current of an open system is given by Eq. (2) with $\sigma=1$ and

$$
b(\epsilon)=-c \frac{\lambda(\epsilon) \kappa(\epsilon)}{2 J_{\infty}(\epsilon)} .
$$

Using the values of $J_{\infty}$ and $\kappa$ obtained in Ref. [10] and $c$ $=3 / 2$ we find

$$
b(\epsilon)=\frac{3}{2} \frac{(2+\epsilon) v+2 \epsilon}{2(v+\epsilon)}, \quad v=\sqrt{\frac{1+\epsilon}{1-\epsilon}}+1 .
$$

In Fig. 2 the coefficient $b(\epsilon)$ is plotted for $0 \leqslant \epsilon<1$. This curve has been verified by direct numerical simulations of the KLS model on an open chain in the maximal current phase, demonstrating that the prefactor $c$ indeed does not depend on $\epsilon$. Using (10) it is readily seen that for $\epsilon>0.8$ the value of $b$ is larger than 2 .

According to the criterion conjectured in Ref. [2] one expects phase separation to take place at high densities in model (3) for $\epsilon>0.8$, as long as $\alpha$ is such that the KLS model is in the maximal current phase. This condensed phase-separated state belongs to case $B$ of the criterion. We have carried out extensive numerical simulations of the dynamics of the model for various values of $\epsilon$. We find that for $\epsilon \lesssim 0.4$ no phase separation is observed. However, for $\epsilon$ $>0.4$ simulation of systems of sizes up to $L=10^{6}$ show that the system evolves towards what seems to be a phaseseparated state at sufficiently large densities. We argue that a genuine phase separation takes place only for $\epsilon>0.8$. On the other hand, the seemingly phase separation found in simulations for $0.4 \leq \epsilon<0.8$, is only a result of large but finite correlation lengths, as was found in the AHR and in the twolane models. As pointed out in Ref. [10] such a behavior is related to corrections of order $1 / \mathrm{n}^{2}$ and higher in the form of the current [Eq. (2)]. These corrections were shown to lead to a crossover with a very sharp increase in the correlation length, which could be erroneously interpreted as a genuine phase transition in numerical studies of finite systems.

We now discuss the phase transition leading to the phaseseparated state. According to Ref. [2] the domain size distribution just below the transition takes the form

$$
\mathcal{P}(n) \sim \frac{1}{n^{b}} e^{-n / \xi},
$$

where $\xi$ is the correlation length, which diverges at the transition. The particle density in the system is related to $\xi$ by $\rho /(1-\rho)=\Sigma n \mathcal{P}(n) / \Sigma \mathcal{P}(n)$. The critical density $\rho_{c}$ is given by this expression with $\xi \rightarrow \infty$. Note that with this form of the distribution function, $\rho_{c}$ is 1 in the limit $b \backslash 2$, and is a decreasing function of $b$. It is straightforward to show [14] that the divergence of the correlation length at the critical density is given by

$$
\xi \sim \begin{cases}\left|\rho-\rho_{c}\right|^{-1 /(b-2)}, & 2<b<3, \\ \left|\rho-\rho_{c}\right|^{-1}, & b>3 .\end{cases}
$$

It is worthwhile noting that while $\partial \xi^{-1} / \partial \rho$ is continuous at the transition for $2<b<3$, it exhibits a discontinuity for $b$ $>3$. The transition may thus be considered continuous for $2<b<3$ and first order for $b>3$.

In the model defined above $b$ is found to satisfy $3 / 2 \leqslant b$ $<9 / 4$. It is natural to ask whether larger values of $b$ could be reached by increasing the range of the interactions. To answer this question we have extended model (4) to include next-nearest-neighbor interactions,

$$
H=-\epsilon / 4 \sum_{i} s_{i} s_{i+1}-\delta / 4 \sum_{i} s_{i} s_{i+2} .
$$

We have calculated the value of $b$ as a function of $\delta$ by Monte Carlo simulations. This is done by measuring the current $J_{n}$ in an open system of size $n$, which is fully occupied by positive and negative particles. At the boundaries, the coupling to the rest of the system is modeled by injection of positive (negative) particles with rates $\alpha$ at the left (right). Simulating systems of size up to 1024 enables us to fit the measured values of $J_{n}$ to the form (2) with $\sigma=1$ and to 


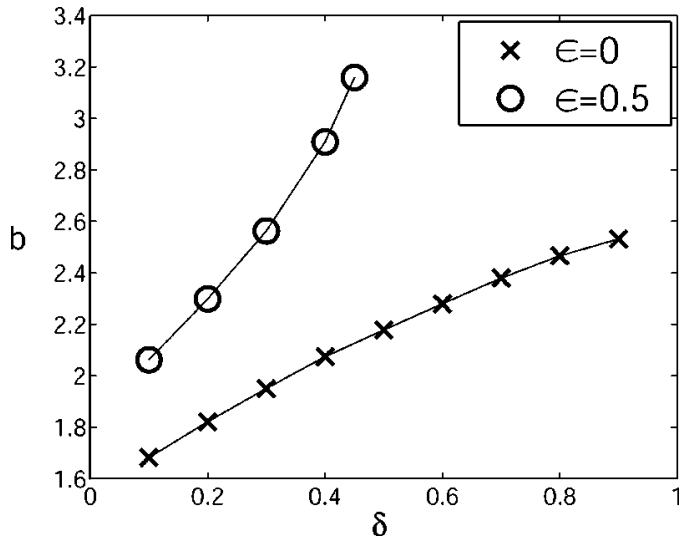

FIG. 3. The coefficient $b(\delta)$, as calculated from Monte Carlo simulations of domains of sizes up to 1024 .

extract $b$. In Fig. 3 we plot $b$ as a function of $\delta$, for $\epsilon=0$ and for $\epsilon=0.5$. We find that by extending the range of the interactions one can increase $b$ to values even larger than 3 , where the phase-separation transition is expected to be first order.

The models introduced in this work exhibit a class $B$ phase separation transition according to the recently conjec- tured criterion [2]. The models exhibit a homogeneous state at low densities and a phase transition into a phase-separated state at a critical density. In these models the macroscopic domain of the condensed phase is composed of a fluctuating mixture of the two types of particles in the systems. However the dynamics of the models is such that vacancies do not enter into the condensed phase and they reside only in the fluid phase.

While the validity of the criterion was proved for the AHR model, its general validity was conjectured based on some plausible assumptions on the behavior of the coarsening domains [2]. It would be of interest to analyze the class of models introduced in the present study by other analytical means, in order to verify the validity of the criterion. It would also be of interest to apply the dynamical picture discussed in this work to jamming transitions occurring in traffic models [15].

We thank L. Gray, D. Kandel, J. L. Lebowitz, and G. Ziv for discussions. The support of the Israeli Science Foundation and the Einstein Center are gratefully acknowledged. R.D.W. acknowledges the Minerva Foundation for support during his stay at the Weizmann Institute.
[1] For recent reviews, see D. Mukamel, in Soft and Fragile Matter: Nonequilibrium Dynamics, Metastability and Flow, edited by M.E. Cates and M.R. Evans (Institute of Physics, Bristol, 2000); G.M. Schüt, in Phase Transitions and Critical Phenomena, edited by C. Domb and J. Lebowitz (Academic, London, 2000), Vol. 19.

[2] Y. Kafri, E. Levine, D. Mukamel, G.M. Schütz, and J. Török, Phys. Rev. Lett. 89, 035702 (2002).

[3] M.R. Evans, Y. Kafri, H.M. Koduvely, and D. Mukamel, Phys. Rev. Lett. 80, 425 (1998); Phys. Rev. E 58, 2764 (1998).

[4] P.F. Arndt, T. Heinzel, and V. Rittenberg, J. Phys. A 31, L45 (1998); J. Stat. Phys. 97, 1 (1999).

[5] R. Lahiri and S. Ramaswamy, Phys. Rev. Lett. 79, 1150 (1997); R. Lahiri, M. Barma, and S. Ramaswamy, Phys. Rev. E 61, 1648 (2000).

[6] N. Rajewsky, T. Sasamoto, and E.R. Speer, Physica A 279, 123 (2000); T. Sasamoto and D. Zagier, J. Phys. A 34, 5033 (2001).

[7] G. Korniss, B. Schmittmann, and R.K.P. Zia, Europhys. Lett.
45, 431 (1999); J.T. Mettetal, B. Schmittmann, and R.K.P. Zia, ibid. 58, 653 (2002).

[8] S. Katz, J.L. Lebowitz, and H. Spohn, J. Stat. Phys. 34, 497 (1984).

[9] J.S. Hager, J. Krug, V. Popkov, and G.M. Schütz, Phys. Rev. E 63, 056110 (2001). Note that the definition of $\epsilon$ differs from ours by a sign.

[10] Y. Kafri, E. Levine, D. Mukamel, and J. Török, J. Phys. A 35, L459 (2002).

[11] H. van Beijeren, R. Kunter, and H. Spohn, Phys. Rev. Lett. 54, 2026 (1985).

[12] J. Krug and P. Meakin, J. Phys. A 23, L987 (1990); J. Krug and L. Tang, Phys. Rev. E 50, 104 (1994).

[13] J. Krug, Adv. Phys. 46, 139 (1997).

[14] Y. Kafri, D. Mukamel, and L. Peliti, Eur. Phys. J. B 27, 135 (2002).

[15] L. Gray and D. Griffeath, J. Stat. Phys. 105, 413 (2001). 